\title{
INTELLIGENT HYBRID FUZZY LOGIC SYSTEM FOR DAMAGE DETECTION OF BEAM-LIKE STRUCTURAL ELEMENTS
}

\author{
Sasmita Sahu, Priyadarshi Biplab Kumar, Dayal R. Parhi \\ Department of Mechanical Engineering, Robotics Lab., NIT Rourkela, India \\ e-mail: gudusasmita@gmail.com; p.biplabkumar@gmail.com; dayalparhi@yahoo.com
}

\begin{abstract}
Fuzzy logic has been used in different research fields for more than three decades. It has become a robust method to solve complex and intricate problems which are otherwise difficult to solve by traditional methods. But it still requires some human experience and knowledge. In the present study, an attempt is made to design a hybrid optimization technique for automatic formation of the fuzzy knowledge based rules using an evolutionary algorithm. This hybridization technique has been applied in the field of damage detection and location of cracks in cracked structural elements. In this paper, a robust fault diagnostic tool based on a differential evolution algorithm and fuzzy logic has been proposed. Theoretical and Finite Element analyzes are done to model the crack and to find the effect of the presence of cracks on changes of vibrational characteristic (natural frequencies) of a fixed-fixed beam. The inputs to DEA-FL system are the first three relative natural frequencies, and the outputs from the system are the relative crack depth and relative crack location. For the validation of the results obtained from the proposed method and to check the robustness of the controller, experimental analysis is performed. To find average error rates, the bootstrap method has been adopted.
\end{abstract}

Keywords: fuzzy logic, differential evolution algorithm, crack, natural frequency

\section{Introduction}

Zadeh (1965) introduced Fuzzy Logic (FL). He introduced a concept of the partial set membership rather than a classical set membership or non-membership. Fuzzy Logic was then presented as a way of processing data and not presented as a control method. Fuzzy Logic is a mathematical logic that attempts to solve problems by assigning values to an imprecise range of data to reach the most accurate result. FL is an approach to compute "degrees of truth" rather than fully "true" or "false" (1 or 0$)$ of the classical logic. Due to its rule over the traditional method, it has been widely used in various fields. Some of researchers (Ganguli, 2001; Jiao et al., 2015) also used this logic system in structural health monitoring, navigation of robots (Parhi, 2005), etc. Fuzzy Logic System (FLS) consists of fuzzy sets, membership functions and rule tables. The main aim of any FLS is first to define the membership functions. The MFs are defined by the parameters based on the author's experience. Some of the researchers have used genetic algorithms to fuzzy logic together, but in this case the results from the genetic algorithm layer is trained again in the fuzzy logic layer for refinement (Dash and Parhi, 2014), some have used hybridized membership functions to be applied in the fuzzy inference system (Thatoi et al., 2014).

The Differential Evolution Algorithm (DEA) was proposed by Storn and Price (1997). The algorithm became popular due to its capacity of producing effective results with simplicity. This is a population oriented evolutionary algorithm (Tang, 2012). It has the capacity of memorising individual's optimal value by sharing the internal information. The operators involved are mutation, crossover and selection (Qin and Suganthan, 2005). Most of the time, it is considered as a greedy Genetic Algorithm while ensuring quality. DE has been successfully applied in diverse 
fields such as mechanical engineering, communication, and pattern recognition (Dewhirst et al., 2010).

Due to the competition to produce better and better results by using Artificial Intelligence (AI) techniques, different researchers have tried to hybridize different AI techniques or improved the performance of the AI techniques by using different enhanced operations. Some of the researchers have tuned parameters of DEA using a memtic algorithm (Neri, 2008), hybridized PSO with DEA (Thangaraj et al., 2010), some have used FLS for diversity control of population of DEA (Amali and Baskar, 2013), and some others have used multi objective evolutionary algorithms for the enhancement of performance of FLC (Xue et al., 2005). But less work has been proposed to integrate FLS and DEA using simple steps to be used in the field of vibration analysis of cracked structures for crack location.

Cracks, faults or damages are a serious threat to the current and future performance of a system. This may occur due to over stressing during operation in extreme environmental conditions or due to any accidental scenarios. The present crack may grow during working and may lead to failure if the crack grows beyond the critical limit. So it is needed to investigate the fault occurrence in structures at the earliest possible stage. Damage detection methods employing vibration characteristics of a component of a structure have emerged as a reliable method for predicting structural health (Khiem and Tran, 2013; Daneshmehr et al., 2013). Damage assessment attempts to determine whether structural damage has occurred try to find the location and extent of the damage (Pawar and Sawant, 2014). The methods using vibration analysis offer some advantages over conventional methods. The frequency measurement method is easier to use in in real-time as it only requires a small number of sensors, and the measurement is straightforward. Furthermore, to make the analysis of nonlinearity in the vibration based fault detection methods, different researchers use the finite element analysis (Sinha et al., 2002; Caddemi and Morassi, 2013). Nowaday, numbers of software packages are available in the market that makes the analysis easier (Musmar et al., 2014; Parandaman and Jayaraman, 2014). This work aims at advancing damage detection methods by proposing this method of crack detection. The results suggest the evidence of presence of a crack. A 2-D finite element model has been developed to study modal properties and identify the presence of a crack in the structural element.

In the process of damage identification and detection, traditional mathematical techniques are rather insufficient due to difficulty in the modelling of highly nonlinear components. New methods of the modelling based on AI techniques have shown promising results for the modelling of nonlinearities. So the inverse problem of damage detection of any material of any section can be solved using computational intelligent techniques. In the effort to find a convenient solution to a problem using AI systems, several difficulties are faced by the designer. It is not an easy task to solve problems while running an algorithm. This clearly paves the way to find better AI systems in a hit and trial method. Due to the lack of a common framework, it remains often difficult to compare various AI systems conceptually and evaluate their performance comparatively. So the results are purely based on the problem definition and the designer when applied to different approaches.

In this paper, a hairline transverse crack is modeled using Finite Element Analysis (FEA). Then the first three natural frequencies are extracted and converted into relative values from FEA. The relative values of the natural frequencies (rfnf, rsnf, rtnf) are found out by comparing the natural frequencies of the uncracked and cracked beam. Relative values of the crack depth and crack location ( $\mathrm{rcd}, \mathrm{rcl}$ ) are also found using a similar method. This work considers only the natural frequencies because they are less prone to error while calculating. The relative first natural frequency (rfnf), second (rsnf) and relative third natural frequency (rtnf) are treated as the input variables in the proposed method. The outputs from the system are the relative values 
of crack depth (rcd) and crack location (rcl) which, in turn, contain the information of damage severity.

\section{Cracked beam modelling}

A mathematical model must be chosen to obtain results by numerical simulation. Most of the studies are made assuming the crack to be open and remain open during vibration. For theoretical and finite element analysis, it is assumed that there is no displacement and rotation of the beam at the clamped end and the crack is a non-propagating crack. Most of the studies are made assuming the crack to be open and remain open during vibration to make the mathematical modeling simple. A fixed-fixed beam with a transverse hairline crack has been modeled in the present work. Due to the presence of the crack, an additional flexibility is introduced which can be defined in a matrix form. In this work, a $2 \times 2$ matrix is considered. The dimension of the matrix depends on the degrees of freedom. The transverse surface crack has depth $a$, width $B$ and height $W$. Here, the fixed-fixed beam is subjected to the axial force $P_{1}$ and the bending moment $P_{2}$ as shown in Fig. 1.

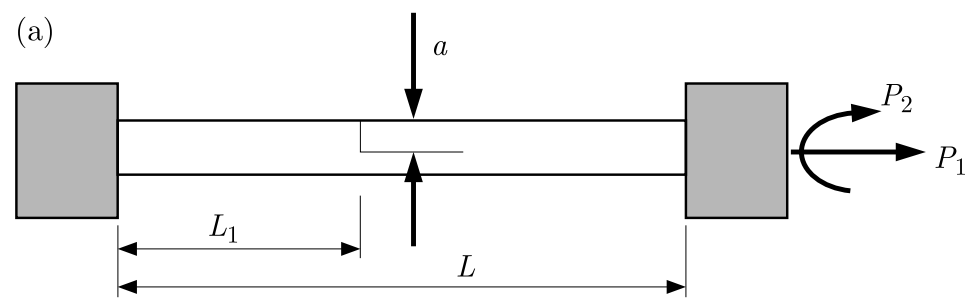

(b)

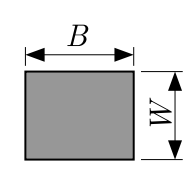

Fig. 1. (a) Cracked fixed-fixed beam, (b) cross sectional view of the cracked fixed-fixed beam

Tada et al. (1973) proposed the strain energy release rate at the fractured section as

$$
J=\frac{1}{E^{\prime}}\left(K_{11}+K_{12}\right)^{2}
$$

where

$$
\text { frac } 1 E^{\prime}= \begin{cases}\frac{1-\gamma^{2}}{E} & \text { for plain strain condition } \\ \frac{1}{E} & \text { for plain stress condition }\end{cases}
$$

(1) where $K_{11}, K_{12}$ are the stress intensity factors of mode I (opening of the crack) for the load $P_{1}$ and $P_{2}$, respectively.

Taking $U_{t}$ as the strain energy due to the crack and applying Castigliano's theorem, the additional displacement along the force $P_{i}$ can be calculated

$$
u_{i}=\frac{\partial U_{t}}{\partial P_{i}}
$$

By definition, the flexibility influence coefficient $C_{i j}$ which forms the elements of the flexibility matrix can be written as

$$
C_{i j}=\frac{\partial u_{i}}{\partial P_{j}}=\frac{\partial^{2}}{\partial P_{i} \partial P_{j}} \int_{0}^{a} J(a) d a
$$


After finding out all the four coefficients of the flexibility matrix, the local stiffness matrix can be obtained by taking the inversion of the compliance matrix, i.e.

$$
\mathbf{K}=\left[\begin{array}{ll}
K_{11} & K_{12} \\
K_{21} & K_{22}
\end{array}\right]=\left[\begin{array}{ll}
C_{11} & C_{12} \\
C_{21} & C_{22}
\end{array}\right]^{-1}
$$

From the governing equations of the free vibration mode of the cracked beam, the normal function for the system can be defined as

$$
\begin{aligned}
& \bar{U}_{1}(\bar{x})=A_{1} \cos \left(\bar{K}_{u} \bar{x}\right)+A_{2} \sin \left(\bar{K}_{u} \bar{x}\right) \quad \bar{U}_{2}(\bar{x})=A_{3} \cos \left(\bar{K}_{u} \bar{x}\right)+A_{4} \sin \left(\bar{K}_{u} \bar{x}\right) \\
& \bar{Y}_{1}(\bar{x})=A_{5} \cosh \left(\bar{K}_{y} \bar{x}\right)+A_{6} \sinh \left(\bar{K}_{y} \bar{x}\right)+A_{7} \cos \left(\bar{K}_{y} \bar{x}\right)+A_{8} \sin \left(\bar{K}_{y} \bar{x}\right) \\
& \bar{Y}_{2}(\bar{x})=A_{9} \cosh \left(\bar{K}_{y} \bar{x}\right)+A_{10} \sinh \left(\bar{K}_{y} \bar{x}\right)+A_{11} \cos \left(\bar{K}_{y} \bar{x}\right)+A_{12} \sin \left(\bar{K}_{y} \bar{x}\right)
\end{aligned}
$$

where

$$
\begin{aligned}
& \bar{x}=\frac{x}{L} \\
& \bar{u}=\frac{u}{L} \\
& \bar{K}_{u}=\frac{\omega L}{C_{u}} \\
& C_{u}=\sqrt{\frac{E}{\rho}} \\
& \bar{y}=\frac{y}{L} \\
& \beta=\frac{L_{1}}{L} \\
& \bar{K}_{u}=L \sqrt{\frac{\omega}{C_{y}}} \\
& C_{y}=\sqrt{\frac{E I}{\mu}} \\
& \mu=A \rho
\end{aligned}
$$

Here $U_{1}(x, t)$ and $U_{2}(x, t)$ are normal functions of longitudinal vibration for the sections before and after the crack and $Y_{1}(x, t), Y_{2}(x, t)$ are normal functions of bending vibration for the same sections.

Analyzing the normal functions of the longitudinal and bending vibration condition, the characteristic equation (the determinant) of the system can be written as

$$
|Q|=0
$$

This determinant $|Q|$ is a function of the natural circular frequency $\omega$, the relative location of the crack $\beta$ and the local stiffness matrix $\mathbf{K}$ which, in turn, is a function of the relative crack depth $a / W$.

\section{Finite element modelling of the cracked beam}

For vibration analysis of the uncracked and cracked cantilever beam, ALGOR V 19.3 SP 2 Finite Element Program is used. First, the beam element with a different single crack is plotted using CATIA V5R15 software, and then they are treated in ALGOR environment. The uncracked and cracked beam model is then analyzed in ALGOR environment. First of all, mesh generation is performed. The mesh size is around $1.4529 \mathrm{~mm}$ and approximately 33369 elements are created. Then the parameters such as the element type (brick and isotropic), material name (aluminium alloy) are defined in the ALGOR environment. After that boundary conditions are given by constraining all degrees of freedom of the nodes located on the left end of the beam. The model unit is then changed to SI standards. Then, in the analysis window, a particular analysis type is selected (natural frequency, i.e. modal analysis). Then the analysis is performed and the first three modes of natural frequencies at different crack locations and crack depths of the fixedfixed beam are recorded. Figure 2 shows the modes of vibration of the cracked beam after finite element analysis.

\section{Definition of Fuzzy Logic System parameters while applied to Fault Detection}

Fuzzy logic is a logic based system in which the fuzzy logic is used to represent different forms of knowledge to model interactions and relationships among system variables. Fuzzy logic systems are a very important tool for the modeling of complex systems. The shortcomings of fuzzy logic 
(a)

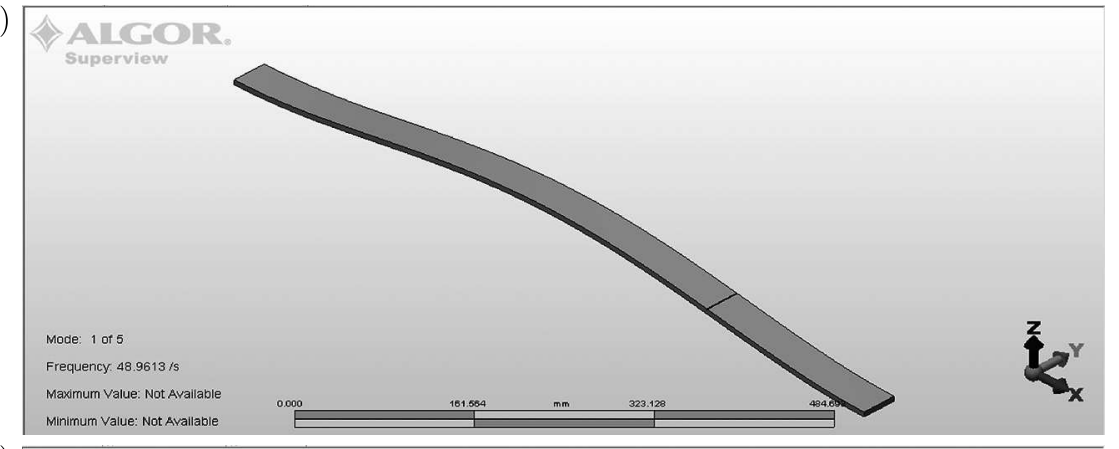

(b)

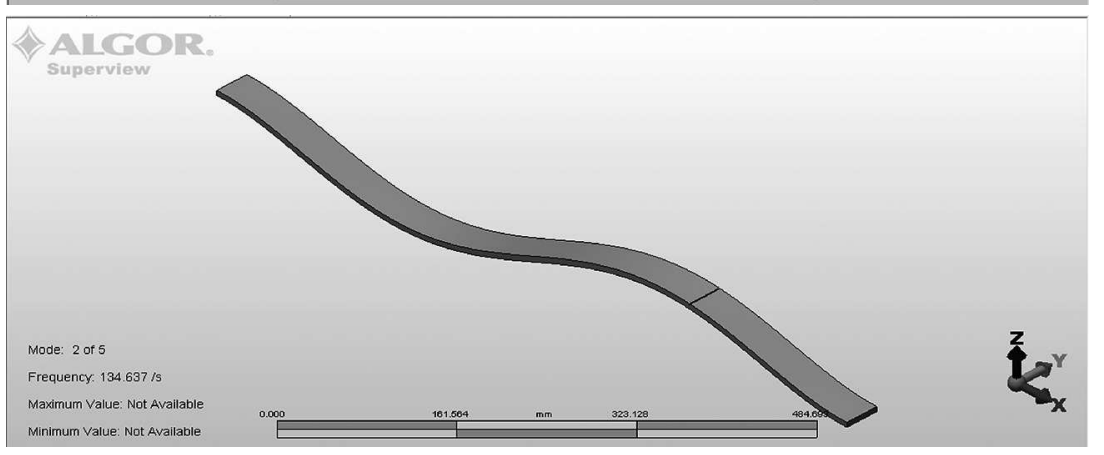

(c)

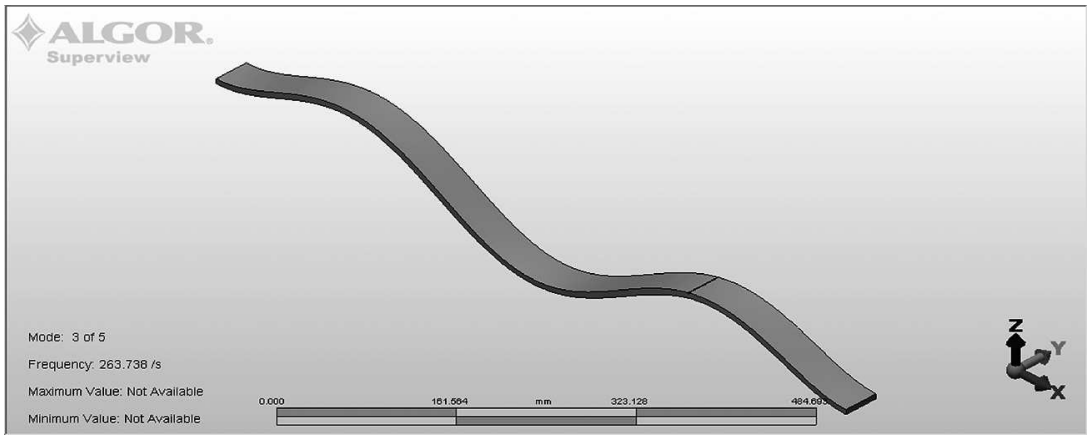

Fig. 2. First three modes of vibration of the cracked fixed-fixed beam

are systematic design and learning capacity. The main challenge of a fuzzy inference system is to design fuzzy rules according to the problem definition.

Another main issue is to design the membership function parameters (variable definition) involved with the rule. From the literature available, it can be observed that many methods have been formulated to tune the MF parameters using different evolutionary algorithms. But less of the work is done to optimize both fuzzy membership functions and fuzzy rules. So in the present work, an effort is made to optimize both the fuzzy membership function and the fuzzy rules.

The proposed fuzzy system is based on the Mamdani fuzzy model (Fig. 3). Fuzzy linguistic variables are defined before assigning different membership functions and definition of their ranges. These linguistic variables play a major role during formation of the rule table. These are linguistic objects or words rather than numbers.

The different linguistic variables used for input and output variables are given as below:

Input 1: (rfnf): low (L), medium (M), high (H)

Input 2: (rsnf): low $(\mathrm{L})$, medium $(\mathrm{M})$, high $(\mathrm{H})$

Input 3: (rtnf): low (L), medium $(\mathrm{M})$, high $(\mathrm{H})$

Output 1: (rcd): small (S), medium (M), large (L)

Output 2: (rcl): small (S), medium (M), big (B) 


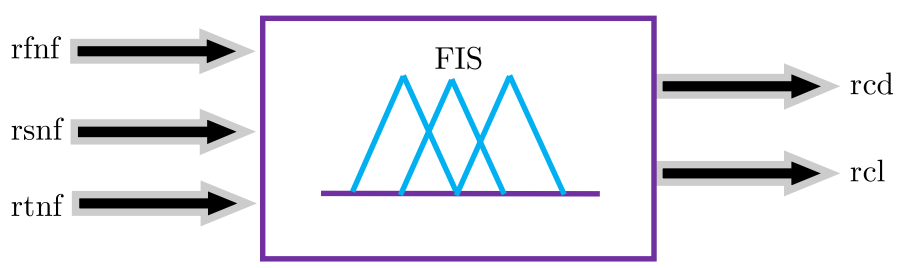

Fig. 3. Fuzzy controller with its input and output variables

The rule base section of the fuzzy inference system works on the application and implementation of the fuzzy rules. The fuzzy rules are usually of the form of "if-then" statements. The if antecedent part and then - part is known as the consequent part. The two fuzzy parts are connected with the connectors like AND, OR, NOT, etc.

A typical rule in a Mamdani fuzzy system in the current problem is defined as below:

If $x_{1}$ is $\mathrm{LF}, x_{2}$ is MF, $x_{3}$ is LF then $y_{1}$ is $\mathrm{SD}, y_{2}$ is ML

Based on the fuzzy subset, the fuzzy rules are defined in a general form as follows

If ( $\mathrm{fnf}$ is $\mathrm{fnf}_{i}$ and $\operatorname{snf}$ is $\operatorname{snf}_{j}$ and $\operatorname{tnf}$ is $\left.\operatorname{tnf}_{k}\right)$ then $\left(\mathrm{cd}\right.$ is $\mathrm{cd}_{i j k}$ and $\mathrm{cl}$ is $\left.\mathrm{cl}_{i j k}\right)$

where $i j, k=1,2,3$, because of "rfnf", "rsnf", "rtnf" have 3 membership functions each.

From above expression (4.2), two sets of rules can be written

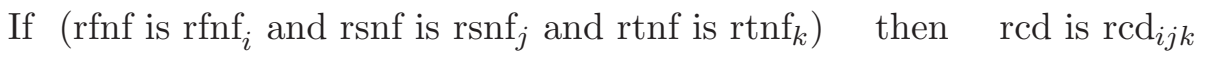

If $\left(\operatorname{rfnf}_{\text {is }} \operatorname{rnff}_{i}\right.$ and $\operatorname{rsnf}$ is $\operatorname{rsnf}_{j}$ and $\operatorname{rtnf}$ is $\left.\operatorname{rtnf}_{k}\right)$ then $\mathrm{rcl}$ is $\operatorname{rcl}_{i j k}$

According to the usual Fuzzy Logic control method (Parhi, 2005), a factor $w_{i j k}$ is defined for the rules as follows

$$
w_{i j k}=\mu_{\text {fnf }_{i}}\left(\text { freq }_{i}\right) \wedge \mu_{\text {snf }_{j}}\left(\text { freq }_{j}\right) \wedge \mu_{\text {tnf }_{k}}\left(\text { freq }_{k}\right)
$$

where $\mathrm{freq}_{i}$, freq $j$ and $\mathrm{freq}_{k}$ are the first, second and third natural frequency of the cantilever beam with a crack, respectively. Applying the composition rule of interference (Parhi, 2005), the membership values of the relative crack location and relative crack depth (location) are given as

$$
\begin{array}{ll}
\mu_{\operatorname{rcl}_{i j k}}(\text { location })=w_{i j k} \wedge \mu_{\operatorname{rcl}_{i j k}} \text { (location) } & \forall \text { length } \in \mathrm{rcl} \\
\mu_{\operatorname{rcd}_{i j k}}(\text { depth })=w_{i j k} \wedge \mu_{\operatorname{rcd}_{i j k}}(\text { depth }) & \forall \text { depth } \in \mathrm{rcd}
\end{array}
$$

The overall conclusion by combining the output can be written as follows

$$
\begin{aligned}
& \mu_{\mathrm{rcl}}(\text { location })=\mu_{\operatorname{rcl}_{11111}}(\text { location }) \vee \ldots \vee \mu_{\operatorname{rcl}_{i j k}}(\text { location }) \vee \ldots \vee \mu_{\operatorname{rcl}_{131313}}(\text { location }) \\
& \mu_{\operatorname{rcd}}(\operatorname{depth})=\mu_{\operatorname{rcd}_{111111}}(\operatorname{depth}) \vee \ldots \vee \mu_{\operatorname{rcd}_{i j k}}(\operatorname{depth}) \vee \ldots \vee \mu_{\operatorname{rcd}_{131313}}(\operatorname{depth})
\end{aligned}
$$

The crisp values of thw relative crack location and relative crack depth are computed using the center of gravity method (Parhi, 2005) as

$$
\begin{aligned}
& \text { Relative crack location }=\mathrm{rcl}=\frac{\int \text { location } \mu_{\mathrm{rcl}}(\text { location }) d \text { location }}{\int \mu_{\mathrm{rcl}}(\text { location }) d \text { location }} \\
& \text { Relative crack depth }=\mathrm{rcd}=\frac{\int \operatorname{depth} \mu_{\mathrm{rcd}}(\text { depth }) d \text { depth }}{\int \mu_{\mathrm{rcd}}(\text { depth }) d \text { depth }}
\end{aligned}
$$




\section{Analysis of fault detection using simple differential evolution algorithm (DEA)}

Differential Evolution (DE) which utilizes $N_{P} D$-dimensional vectors of real valued parameters is a parallel direct search method. $P(G)$ is the current population composed of encoded with individuals $X_{i}$. Figure 4 describes the idea of the Differential Evolution Algorithm which has been used for crack detection in the current research work.

Here $X_{i}=\left\{x_{1}, x_{2}, x_{3}, x_{4}, x_{5}\right\}$, where $x_{1}-\operatorname{rfnf}, x_{2}-\operatorname{rsnf}, x_{3}-\operatorname{rtnf}, x_{4}-\operatorname{rcd}, x_{5}-\mathrm{rcl}$, $G$ - number of generations, $D$ - number of parameters.

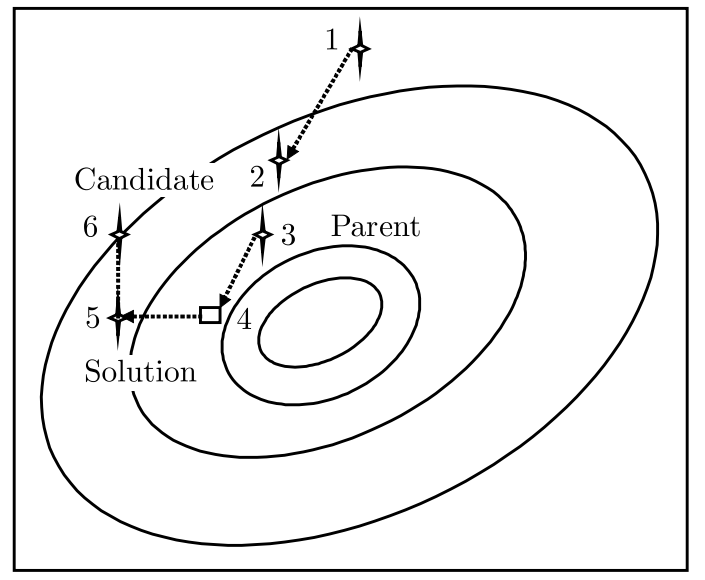

Fig. 4. Differential evolution algorithm

The following steps describe the algorithm for Differential Evolution Algorithm.

1. Read the parameters - scaling factor $F$, crossover constant $C_{r}$, population size $N_{p}$, maximum iterations $G_{\max }$ and decision variables $D$ (i.e. $\mathrm{rfnf}$, rsnf, rtnf, rcd and $\mathrm{rcl}$ ).

2. Set the iteration $G=0$, population index $i=1$, set the decision variable $j=1$.

3. Initialise the parent vector uniformly in the random search space. The initial value of the $j$-th parameter in the $i$-th individual at the generation $G=0$ is given as

$$
x_{j, i}^{(0)}=x_{j}^{\min }+\operatorname{rand}_{j}(0,1)\left(x_{j}^{\max }-x_{j}^{\min }\right) \quad j=1, \ldots, D \quad i=1, \ldots, N_{p}
$$

4. Once initialised, DE mutates the population to produce a population of $N_{p}$ mutant vectors

$$
X_{i}^{\prime(G)}=X_{a}^{G}+F\left(X_{b}^{G}-X_{c}^{G}\right) \quad i=1, \ldots, N_{p}
$$

The indices $a, b$ and $c$ are randomly chosen. These indices are different from each other and from the base vector index $i$. The scaling factor $F$ is a real and constant factor $F \in[0,2]$. As this constant is used in the mutation operator and used to control the rate at which the population evolves, here in the current paper it is taken as 0.3.

5. After mutation, uniform crossover is employed to generate trial vectors $X_{i}^{\prime \prime}$ by mixing the mutant vectors and target vectors $X_{i}$

$$
X_{j, i}^{\prime \prime}(G)= \begin{cases}X_{j, i}^{\prime(G)} & \text { if } \operatorname{rand}_{j}(0,1) \leqslant C_{r} \vee j=j_{\text {rand }} \\ X_{j, i}^{(G)} & \text { otherwise }\end{cases}
$$

The crossover operation is applied to each pair of the target vector $X_{i}$ and its corresponding mutant vector $X_{i}^{\prime}$ to generate the trial vector $X_{i}^{\prime \prime}$.

The crossover probability is a user defined value. The crossover probability is defined as $C_{r} \in(0,1)$ and in this work it is taken as 0.6. The crossover operator copies the $j$-th parameter of the mutant vector $X_{i}^{\prime}$ to the corresponding parameter of the trial vector $X_{i}^{\prime \prime}$ if $\operatorname{rand}_{j}(0,1) \leqslant C_{r}$, otherwise it is copied from the corresponding target vector $X_{i}$. 
6. Sometimes the upper and lower bounds of the newly generated trial vectors exceed the given value and then they are randomly and uniformly initialised to the initial value given previously. The objective function values of all trial vectors are evaluated. Then the selection operator (according to the fitness rank) determines the population by choosing between the trial vectors and their predecessors (target vectors):

a) if the trial vector $X_{i}^{\prime \prime}(G)$ has an equal or lower fitness function value (optimal) than that of its target vector $X_{i}^{(G)}$, it replaces the target vector in the next generation,

b) otherwise, the target vector retains its place in the population for at least one more generation

$$
X_{i}^{(G+1)}= \begin{cases}X_{i}^{\prime \prime}(G) & \text { if } f\left(X_{i}^{\prime \prime}(G)\right) \leqslant f\left(X_{i}^{\prime \prime}(G)\right) \quad i=1, \ldots, N_{p} \\ X_{i}^{(G)} & \text { otherwise }\end{cases}
$$

7. Once the new population is installed, the process of mutation, crossover and selection is repeated for several generations.

8. The algorithm stops after reaching threshold values for the target vector.

The hybridized methodology consists of three major steps, i.e., preprocessing, processing, postprocessing. In the preprocessing, the individual solutions are fed to the DEA. Then the fuzzy rules using DEA in this step are fed to the Fuzzy Inference System (FIS) for the implementation and aggregation of the fuzzy rules. In the postprocessing, the results from the FIS are defuzzified to give the crisp result ( $\mathrm{rcd}, \mathrm{rcl}$ ) from the methodology. Figure 5 describes the proposed methodology in the pictorial form.

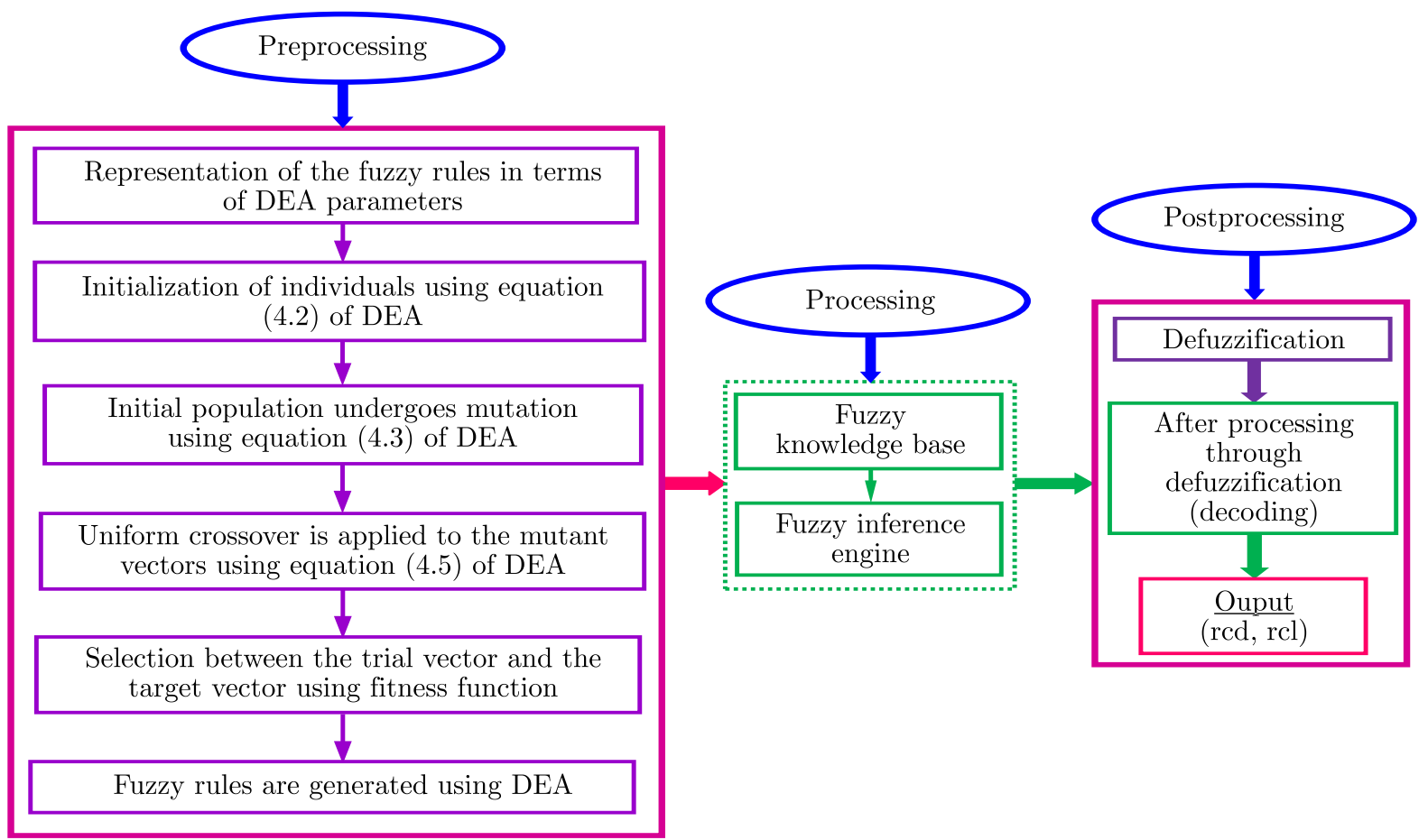

Fig. 5. Pictorial presentation of DEAFL system

Following are the steps used for computation of MF using Differential Evolution Algorithm (DEA) described in detail.

1. In the first step of hybridisation of the DEA and FLS is to represent the fuzzy rules in terms of DEA parameters. 
Here $X_{i}$, which is an individual in the population of DEA, represents the fuzzy rule, where $x_{1}, x_{2}, x_{3}, x_{4}, x_{5}$ represent the linguistic variables of fuzzy membership functions in the fuzzy rule.

2. Then initialisation takes place using equation (5.1) of DEA.

3. The initial population undergoes mutation using equation (5.2) of DEA.

4. Then uniform crossover is applied to the mutant vectors using equation (5.3) of DEA.

5. A fitness function is used to make selection between the trial vector and the target vector, whichever becomes the best fit replaces the target vector in the next generation. Otherwise, if the stopping criteria are met, the algorithm stops making the current target vector as the best solution (fuzzy rule).

6. Likewise when some (Musmar et al., 2014) fuzzy rules are generated using DEA, they are fed to the Mamdani fuzzy inference system.

7. After the implementation and aggregation of the fuzzy rules in the inference system, the results are defuzzified using centroid defuzzification.

8. The defuzzification process shows the crisp output from the fuzzy inference system.

\section{Experimental set-up used in the fault detection of the cracked beam}

The instruments used in free vibration analysis of the fixed-fixed beam are an impact hammer, vibration pick-up, vibration analyzer and vibration indicator. Using the impact hammer, the cracked fixed-fixed beam is excited in the free vibration mode. The vibration analyzer is PULSE LAB Prolite 3560. The excitation parameters are picked up by the vibration pick-up or accelerometer. Then these parameters are fed to the vibration analyzer, where the parameters are analyzed, and the results are shown in the vibration indicator.

Several tests are conducted using the experimental setup (Fig. 6) on aluminium alloy beam specimens $(800 \mathrm{~mm} \times 38 \mathrm{~mm} \times 6 \mathrm{~mm})$ with a transverse crack for determining the natural frequencies at different crack locations and crack depths. These specimens are given vibration by the impact hammer, and the 1st, 2nd and 3rd natural frequencies are recorded in the vibration indicator. The schematic diagram of the experimental set-up is shown in Fig. 7.

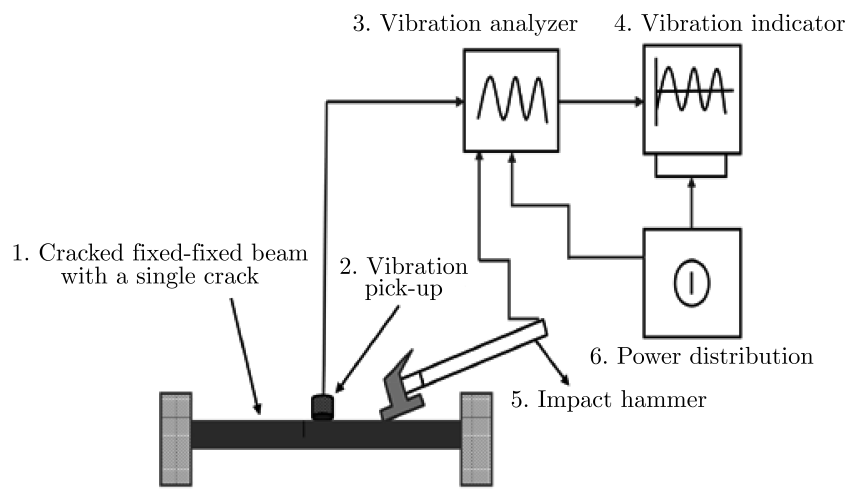

Fig. 6. Schematic diagram of experimental set-up; 1 - cracked fixed-fixed beam with a single crack, 2 - vibration pick-up, 3 - vibration analyzer, 4 - vibration indicator, 5 - impact hammer, 6 - power distribution 


\section{Results and discussion}

The results found from the application of the algorithms are given in this Section. The formula used for calculation of the errors is given in Eqs. (7.1) and (7.2).

The percentage error in FEA is calculated using the following formula

FEA result - Result from the proposed technique

$$
\text { FEAresult } \cdot 100
$$

The percentage error in the experimental work is calculated using the following formula

Exp. result - Result from the proposed technique

$$
\text { Exp.result } \cdot 100
$$

Table 1. Comparison of the results from FLS and FEA

\begin{tabular}{|c|c|c|c|c|c|c|c|c|c|}
\hline No. & $\begin{array}{c}\text { rfnf } \\
\text { from }\end{array}$ & $\begin{array}{c}\text { rsnf } \\
\text { from }\end{array}$ & $\begin{array}{c}\text { rtnf } \\
\text { from }\end{array}$ & $\begin{array}{c}\text { red } \\
\text { from }\end{array}$ & $\begin{array}{c}\text { rcl } \\
\text { from }\end{array}$ & $\begin{array}{c}\text { red using } \\
\text { the FLS } \\
\text { FEA }\end{array}$ & $\begin{array}{c}\text { rcl using } \\
\text { the FLS }\end{array}$ & $\begin{array}{c}\text { percent. } \\
\text { error } \\
\text { FEA }\end{array}$ & $\begin{array}{c}\text { Fercent. } \\
\text { error } \\
\text { rcl }\end{array}$ \\
\hline \hline 1 & 0.9952 & 0.9992 & 0.9944 & 0.28125 & 0.5625 & 0.272194 & 0.543206 & 3.22 & 3.43 \\
\hline 2 & 0.9958 & 0.9998 & 0.9940 & 0.25 & 0.46875 & 0.241275 & 0.451922 & 3.49 & 3.59 \\
\hline 3 & 0.9960 & 0.9999 & 0.9987 & 0.25 & 0.25 & 0.2412 & 0.240325 & 3.52 & 3.87 \\
\hline 4 & 0.9969 & 0.9969 & 0.9952 & 0.1875 & 0.5 & 0.181106 & 0.4817 & 3.41 & 3.66 \\
\hline 5 & 0.9705 & 0.9703 & 0.9700 & 0.2166 & 0.5 & 0.209301 & 0.4811 & 3.37 & 3.78 \\
\hline
\end{tabular}

\begin{tabular}{|c|c|c|c|c|c|c|c|c|c|}
\hline No. & $\begin{array}{l}\text { rfnf } \\
\text { from } \\
\text { FEA } \\
\end{array}$ & $\begin{array}{l}\text { rsnf } \\
\text { from } \\
\text { FEA } \\
\end{array}$ & $\begin{array}{l}\text { rtnf } \\
\text { from } \\
\text { FEA } \\
\end{array}$ & $\begin{array}{l}\text { red } \\
\text { from } \\
\text { FEA } \\
\end{array}$ & $\begin{array}{l}\text { rcl } \\
\text { from } \\
\text { FEA } \\
\end{array}$ & $\begin{array}{l}\text { red using } \\
\text { the FLS } \\
\text { technique }\end{array}$ & $\begin{array}{l}\text { rcl using } \\
\text { the FLS } \\
\text { technique }\end{array}$ & $\begin{array}{c}\text { percent. } \\
\text { error } \\
\text { rcd } \\
\end{array}$ & $\begin{array}{l}\text { percent. } \\
\text { error } \\
\text { rcl } \\
\end{array}$ \\
\hline 1 & 0.9988 & 0.9993 & 0.9976 & 0.15625 & 0.3125 & 0.150703 & 0.300438 & 3.55 & 3.86 \\
\hline 2 & 0.99979 & 0.9960 & 0.9996 & 0.333 & 0.3125 & 0.320712 & 0.300156 & 3.69 & 3.95 \\
\hline 3 & 0.9552 & 0.9551 & 0.9549 & 0.2833 & 0.375 & 0.272025 & 0.362138 & 3.98 & 3.43 \\
\hline 4 & 0.9211 & 0.9219 & 0.9218 & 0.4 & 0.25 & 0.38436 & 0.24055 & 3.91 & 3.78 \\
\hline 5 & 0.9463 & 0.9460 & 0.9458 & 0.367 & 0.3125 & 0.353127 & 0.300969 & 3.78 & 3.69 \\
\hline
\end{tabular}

Table 2. Comparison of the results from FLS and experimental analysis

Table 3. Comparison of the results from DEAFLS and FEA

\begin{tabular}{|c|c|c|c|c|c|c|c|c|c|}
\hline No. & $\begin{array}{c}\text { rfnf } \\
\text { from }\end{array}$ & $\begin{array}{c}\text { rsnf } \\
\text { from }\end{array}$ & $\begin{array}{c}\text { rtnf } \\
\text { from }\end{array}$ & $\begin{array}{c}\text { red } \\
\text { from }\end{array}$ & $\begin{array}{c}\text { rcl } \\
\text { from }\end{array}$ & $\begin{array}{c}\text { red using } \\
\text { the FLS } \\
\text { technique }\end{array}$ & $\begin{array}{c}\text { rcl using } \\
\text { the FLS } \\
\text { technique }\end{array}$ & $\begin{array}{c}\text { percent. } \\
\text { error } \\
\text { rcd }\end{array}$ & $\begin{array}{c}\text { percent. } \\
\text { error } \\
\text { rcl }\end{array}$ \\
\hline \hline 1 & 0.9952 & 0.9992 & 0.9944 & 0.28125 & 0.5625 & 0.274753 & 0.549338 & 2.31 & 2.34 \\
\hline 2 & 0.9958 & 0.9998 & 0.9940 & 0.25 & 0.46875 & 0.24365 & 0.458531 & 2.54 & 2.18 \\
\hline 3 & 0.9960 & 0.9999 & 0.9987 & 0.25 & 0.25 & 0.2444 & 0.24395 & 2.24 & 2.42 \\
\hline 4 & 0.9969 & 0.9969 & 0.9952 & 0.1875 & 0.5 & 0.183544 & 0.4871 & 2.11 & 2.58 \\
\hline 5 & 0.9705 & 0.9703 & 0.9700 & 0.2166 & 0.5 & 0.211467 & 0.48635 & 2.37 & 2.73 \\
\hline
\end{tabular}

In this paper, the bootstrap method has been adapted to find the average error rates. The bootstrap method is a statistical method for obtaining an estimate of the error. The results of the methods with $95 \%$ confidence intervals are provided in Table 5 . As the confidence interval is taken as $95 \%$, the formula for determination of the average error rates is

$$
\mu-1.96 \frac{s}{\sqrt{n}}<\text { mean error }<\mu+1.96 \frac{s}{\sqrt{n}}
$$

where $\mu$ - mean, $s$ - standard deviation, $n$ - sample population. 
Table 4. Comparison of the results from DEAFLS and Experimental Analysis

\begin{tabular}{|c|c|c|c|c|c|c|c|c|c|}
\hline No. & $\begin{array}{c}\text { rfnf } \\
\text { from }\end{array}$ & $\begin{array}{c}\text { rsnf } \\
\text { from }\end{array}$ & $\begin{array}{c}\text { rtnf } \\
\text { from }\end{array}$ & $\begin{array}{c}\text { red } \\
\text { from }\end{array}$ & $\begin{array}{c}\text { rcl } \\
\text { from }\end{array}$ & $\begin{array}{c}\text { red using } \\
\text { the FLS }\end{array}$ & $\begin{array}{c}\text { rcl using } \\
\text { the FLS }\end{array}$ & $\begin{array}{c}\text { percent. } \\
\text { error } \\
\text { rcd }\end{array}$ & $\begin{array}{c}\text { percent. } \\
\text { error } \\
\text { rcl }\end{array}$ \\
\hline \hline 1 & 0.9988 & 0.9993 & 0.9976 & 0.15625 & 0.3125 & 0.152469 & 0.304563 & 2.42 & 2.54 \\
\hline 2 & 0.99979 & 0.9960 & 0.9996 & 0.333 & 0.3125 & 0.324375 & 0.303781 & 2.59 & 2.79 \\
\hline 3 & 0.9552 & 0.9551 & 0.9549 & 0.2833 & 0.375 & 0.275594 & 0.366563 & 2.72 & 2.25 \\
\hline 4 & 0.9211 & 0.9219 & 0.9218 & 0.4 & 0.25 & 0.38936 & 0.242825 & 2.66 & 2.87 \\
\hline 5 & 0.9463 & 0.9460 & 0.9458 & 0.367 & 0.3125 & 0.356247 & 0.303688 & 2.93 & 2.82 \\
\hline
\end{tabular}

Table 5. Results from the bootstrap method with $95 \%$ confidence intervals

\begin{tabular}{|c|c|c|c|c|c|c|c|}
\hline \multicolumn{2}{|c|}{ Table 1 } & \multicolumn{2}{c|}{ Table 2 } & \multicolumn{2}{c|}{ Table 3 } & \multicolumn{2}{c|}{ Table 4 } \\
\hline rcd & rcl & rcd & rcl & rcd & rcl & rcd & rcl \\
\hline \hline $3.298398-$ & $3.516654-$ & $3.631526-$ & $3.567417-$ & $2.174495-$ & $2.263439-$ & $2.500648-$ & $2.426724-$ \\
3.505602 & 3.815346 & 3.932474 & 3.916583 & 2.453505 & 2.636561 & 2.827352 & 2.881276 \\
\hline
\end{tabular}

The performance of the Fuzzy Logic Controller depends on the fuzzy membership functions and fuzzy rules. So, it is very much needed to optimize or adjust the parameters according to the problem domain. Fuzzy rules are usually generated using linguistic terms in the if-then format, and it largely depends on the human expertise to derive them. In all conditions, the correct choice of the fuzzy membership function with the linguistic variables plays an important role in the performance of the Fuzzy Logic Controller.

It is very difficult to present the expert's knowledge perfectly through the linguistic variables, always. The rule base of the Fuzzy Logic Controller has many parameters which must be adjusted. These parameters are capable to alter or modify the controller performance.

The previous work proposed by different researchers (automatic design of the fuzzy membership function) still required human experts to handle the control system. In those works, only the membership functions were optimized. Fuzzy rules which form the skeleton of the fuzzy rule based system still needs human expertise for its generation. So, in order to overcome the above stated problems, a method to produce fuzzy rules automatically is proposed in this work.

\section{Conclusion}

A method for crack prediction in beam-like structures has been designed and developed using a clonal selection algorithm and fuzzy logic. It is found that the presence of cracks has a remarkable effect on the dynamic characteristics of the beam under consideration. Theoretical, finite element and experimental analysis have been carried out to calculate vibration parameters. These vibration parameters have been used to make a database and subsequent design of the hybrid system. Different operations like crossover and mutation used in the clonal selection algorithm are given in detail. Likewise, different parameters of the fuzzy logic are also discussed.

Table 1 and 3 provide a comparison of the results from FEA with the results from FLS and DEAFLS, respectively. Similarly Table 2 and 4 provide a comparison of the results of the experimental analysis with FLS and DEAFLS, respectively.

The error for FLS is found to be $3.402 \%$ and $3.666 \%$ in comparison with the results from FEA for rcd and rcl. In comparison with the results of the experimental analysis for rcd and rcl, the errors are found to be $3.782 \%$ and $3.742 \%$. While applying DEAFLS to the current problem, the errors are found to be $2.314 \%$ and $2.45 \%$ in comparison with the results from FEA for rcd and rcl. While comparing with the results of the experimental analysis for rcd and rcl, the errors are 
found to be $2.664 \%$ and $2.654 \%$, respectively. From the results and errors, it can be concluded that the proposed method of fusion of the fuzzy logic system and the differential evolution algorithm gives better results than the logic system standalone. This improvement occurs due to some automation of generation of fuzzy rules using the differential evolution algorithm. So, this method can be used as a robust tool for online damage detection of cracked structures as well as other engineering applications.

\section{References}

1. Amali S.M.J., Baskar S., 2013, Fuzzy logic-based diversity-controlled self-adaptive differential evolution, Engineering Optimization, 45, 8,899-915

2. Caddemi S., Morassi A., 2013, Multi-cracked Euler-Bernoulli beams: mathematical modeling and exact solutions, International Journal of Solids and Structures, 50, 944-956

3. Daneshmehr A.R., Nateghi A., Inman D.J., 2013, Free vibration analysis of cracked composite beams subjected to coupled bending-torsion loads based on a first order shear deformation theory, Applied Mathematical Modelling, 1-18

4. Dash A.K., Parhi D.R., 2014, Analysis of an intelligent hybrid system for fault diagnosis in cracked structure, Arabian Journal for Science and Engineering, 39, 1337-1357

5. Dewhirst O.P., Simpson D.M., Angarita N., Allen R., 2010, Wiener-Hammerstein parameter estimation using differential evolution, International Conference on Bio-inspired Systems and Signal Processing, 271-276

6. Ganguli R., 2001, A fuzzy logic system for ground based structural health monitoring of a helicopter rotor using modal data, Journal of Intelligent Material Systems and Structures, 12, 397-407

7. Jiao Y.B., Liu H.B., Cheng Y.C., Gong Y.F., 2015, Damage identification of bridge based on Chebyshev polynomial fitting and fuzzy logic without considering baseline model parameters, Shock and Vibration, 1-10

8. Khiem N.T., Tran H.T., 2013, A procedure for multiple crack identification in beam-like structures from natural vibration mode, Journal of Vibration and Control, 1-11

9. Musmar M.A., Rjoub M.I., Hadi M.A.A., 2014, Nonlinear finite element analysis of shallow reinforced concrete beams using solid 65 element, ARPN Journal of Engineering and Applied Sciences, 9, 2, 85-89

10. NERI F., 2008, On memetic differential evolution frameworks: a study of advantages and limitations in hybridization, IEEE, 2135-2142

11. Parandaman P., Jayaraman M., 2014, Finite element analysis of reinforced concrete beam retrofitted with different fiber composites, Middle-East Journal of Scientific Research, 22, 7, 948-953

12. Parhi D.R., 2005, Navigation of mobile robot using a fuzzy logic controller, Journal of Intelligent and Robotic Systems: Theory and Applications, 42, 3, 253-273

13. PaWAR R.S., SaWant S.H., 2014, An overview of vibration analysis of cracked cantilever beam with non-linear parameters and harmonic excitations, International Journal of Innovative Technology and Exploring Engineering, 8, 3, 53-55

14. Qin A.K., Suganthan P.N., 2005, Self-adaptive differential evolution algorithm for numerical optimization, IEEE Explore 2005, 1785-1791

15. Sinha J.K., Friswell M.I., Edwards S., 2002, Simplified models for the location of cracks in beam structures using measured vibration data, Journal of Sound and Vibration, 251, 1, 13-38

16. Storn R., Price K., 1997, Differential evolution - a simple and efficient heuristic for global optimization over continuous spaces, Journal of Global Optimization, 11, 341-359 
17. Tada H., Paris P.C., Irwin G.R., 1973, The Stress Analysis of Cracks Handbook, Del. Research Corporation, Hellertown

18. TAng Y., 2012, Parameter estimation of Wiener model using differential evolution algorithm, International Journal of Circuits, Systems and Signal Processing, 6, 5, 315-323

19. Thangaraj R., Pant M., Abraham A., Bouvry P., 2010, Particle swarm optimization: hybridization perspectives and experimental illustrations, Applied Mathematics and Computation, $1-19$

20. Thatoi D.N., Choudhury S., Jena P.K., 2014, Fault diagnosis of beam-like structure using modified fuzzy technique, Advances in Acoustics and Vibration, 1-18

21. Xue F., Sanderson A.C., Bonissone P.P., Graves R.J., 2005, Fuzzy logic controlled multiobjective differential evolution, IEEE Conference on Fuzzy Systems, 720-725

22. ZADEH L.A., 1965, Fuzzy sets, Information and Control, 8, 338-353

Manuscript received June 13, 2016; accepted for print October 12, 2016 